\title{
Second SNPP Cal/Val campaign: environmental data retrieval analysis
}

\author{
Daniel K. Zhou* ${ }^{\mathrm{a}}$, Allen M. Larar ${ }^{\mathrm{a}}$, Xu Liu ${ }^{\mathrm{a}}$, Jialin Tian ${ }^{\mathrm{a}}$, William L. Smith ${ }^{\mathrm{b}}$, \\ Susan H. Kizer ${ }^{b}$, and Mitch D. Goldberg ${ }^{c}$ \\ ${ }^{a}$ Science Directorate, NASA Langley Research Center, Hampton, VA, USA \\ ${ }^{\mathrm{b}}$ Science Systems and Applications, Inc., Hampton, VA, USA \\ 'NOAA/NESDIS, College Park, MD, USA
}

\begin{abstract}
Satellite ultraspectral infrared sensors provide key data records essential for weather forecasting and climate change science. The Suomi National Polar-orbiting Partnership (Soumi NPP) satellite Environmental Data Records (EDRs) are retrieved from calibrated ultraspectral radiance or Sensor Data Records (SDRs). Understanding the accuracy of retrieved EDRs is critical. The second Suomi NPP Calibration/Validation field campaign was conducted during March 2015 with flights over Greenland. The NASA high-altitude ER-2 aircraft carrying ultraspectral interferometer sounders such as the National Airborne Sounder Testbed-Interferometer (NAST-I) flew under the Suomi NPP satellite that carries the Crosstrack Infrared Sounder (CrIS) and the Advanced Technology Microwave Sounder (ATMS). Herein we inter-compare the EDRs produced from different retrieval algorithms employed on these satellite and aircraft campaign data. The available radiosonde measurements together with the European Centre for Medium-Range Weather Forecasts (ECMWF) analyses are used to assess atmospheric temperature and moisture retrievals from the aircraft and satellite platforms. Preliminary results of this experiment under a winter, Arctic environment are presented.
\end{abstract}

Keywords: Remote sensing, hyperspectral, ultraspectral, inversion, temperature, moisture, validation.

\section{INTRODUCTION}

Atmospheric and surface thermodynamic parameters retrieved from advanced ultraspectral remote sensors aboard satellites are critical to general atmospheric and Earth science research, climate monitoring, and weather prediction. Satellite ultraspectral radiance data have been shown to be significant observations for conducting atmospheric research and monitoring of the Earth's environment. The National Airborne Sounder Testbed-Interferometer (NAST-I) has flown on NASA high-altitude aircraft such as the ER-2, WB-57, and Proteus, and has been collecting infrared ultraspectral data since 1998. NAST-I, maintained and deployed internationally by NASA's Langley Research Center, serves as an ideal validation sensor. Detailed descriptions of the NAST-I instrumentation, data processing methodologies, and data products can be found elsewhere [1]. NAST-I was designed to provide radiometric measurements similar to those obtained from satellite sensors such as the Atmospheric Infrared Sounder (AIRS) [2-3] on the Aqua (2002), the Interferometer Atmospheric Sounding Instrument (IASI) [4-5] on the Meteorological Operational (MetOp-A 2006 and MetOp-B 2012), and the Cross-track Infrared Sounder (CrIS) on the Suomi NPP (2011) satellites [6-8]. NAST-I data obtained during international aircraft campaigns, such as the European Aqua Thermodynamic Experiment (EAQUATE) [9-11] and the Joint Airborne IASI Validation Experiment (JAIVEx) [12-14], have proven to be useful in earlier AIRS and IASI validation studies. The first Suomi NPP Cal/Val campaign was conducted during May 2013. The NASA high altitude ER-2 aircraft was deployed from NASA's Armstrong Flight Research Center in Palmdale, CA. The EDRs produced with different retrieval algorithms using SDRs measured from satellite and aircraft sensors have been intercompared. The available dropsonde and radiosonde measurements together with the European Centre for Medium-Range Weather Forecasts (ECMWF) analysis are used to assess the results of that experiment [15].

*daniel.k.zhou@nasa.gov, Tel: 757-864-5663 
It is critical to understand the accuracy of retrieved EDRs, which is dependent on SDR uncertainty (e.g., instrument random noise and absolute accuracy), ill-posed retrieval system error, and radiative transfer model uncertainty. Detailed sounding retrieval error budget analysis has indicated that major error sources are from having an ill-posed retrieval system and radiative transfer model uncertainty [16]. There are few approaches to validate EDR products. Some common methods rely on radiosonde measurements, ground-based measurements, and dedicated airborne campaigns providing in-situ measurements of the atmosphere and/or employing similar ultraspectral interferometer sounders aboard aircraft. Ultraspectral interferometer sounders aboard aircraft measure SDRs to retrieve EDR products, which can be used to validate similar products from satellite measurements.

Retrieval accuracy varies with the atmospheric condition and/or environment being assessed. In order to validate satellite EDR performance under a variety of atmospheric conditions, aircraft field campaigns need to be conducted more often in different geophysical locations, and for different atmospheric and surface environments. The second Suomi NPP Cal/Val campaign, conducted in March 2015 over Greenland, provides data for Suomi NPP CrIS/ATMS measurements evaluation for a winter, Arctic environment. This paper presents atmospheric profile retrieval results from NAST-I measurements obtained during the second Suomi NPP Cal/Val Campaign, together with the European Centre for Medium-Range Weather Forecasts (ECMWF) analyses and available radiosonde measurements to evaluate NOAA CrIS/ATMS operational EDR products obtained from the current operational retrieval system, the NOAA Unique CrIS/ATMS Processing System (NUCAPS). At some point later, the CrIS retrieval system may be called NUCALS. An overview of the second Suomi NPP Cal/Val field campaign and the validation implementation and approach followed for assessing and inter-comparing retrievals from both aircraft and spacecraft are discussed herein, results are presented in Section 2, and this is followed by conclusions drawn from them in Section 3.

\section{SECOND SUOMI NPP CAL/VAL CAMPAIGN AND RETRIEVAL VALIDATION}

The second Suomi NPP Cal/Val campaign was conducted during March 2015. The NASA high altitude ER-2 aircraft was deployed from Keflavik Airport, Reykjavík, Iceland. It carried the NAST-I (ultraspectral interferometer sounder) and NAST-M (microwave sounder), the Scanning High-resolution Interferometer Sounder (S-HIS), and the MODIS/ASTER (MASTER) airborne simulator. The ER-2/NAST-I high horizontal resolution retrievals (2.5-km FOV) have shown the atmospheric and surface variations observed from nearby ocean to Greenland during a winter, Arctic environment. A Planetary Boundary Layer (PBL) temperature inversion was captured over the Greenland ice-sheet where the surface was extremely cold. As seen in the Figure 1, in the area within an approximately $400-\mathrm{km}$ track, the atmospheric temperature variation could be as large as $15-20^{\circ} \mathrm{K}$ from ocean to land. The NAST-I retrieval is suitable for satellite retrieval evaluation as an independent measurement and independent retrieval methodology employed.
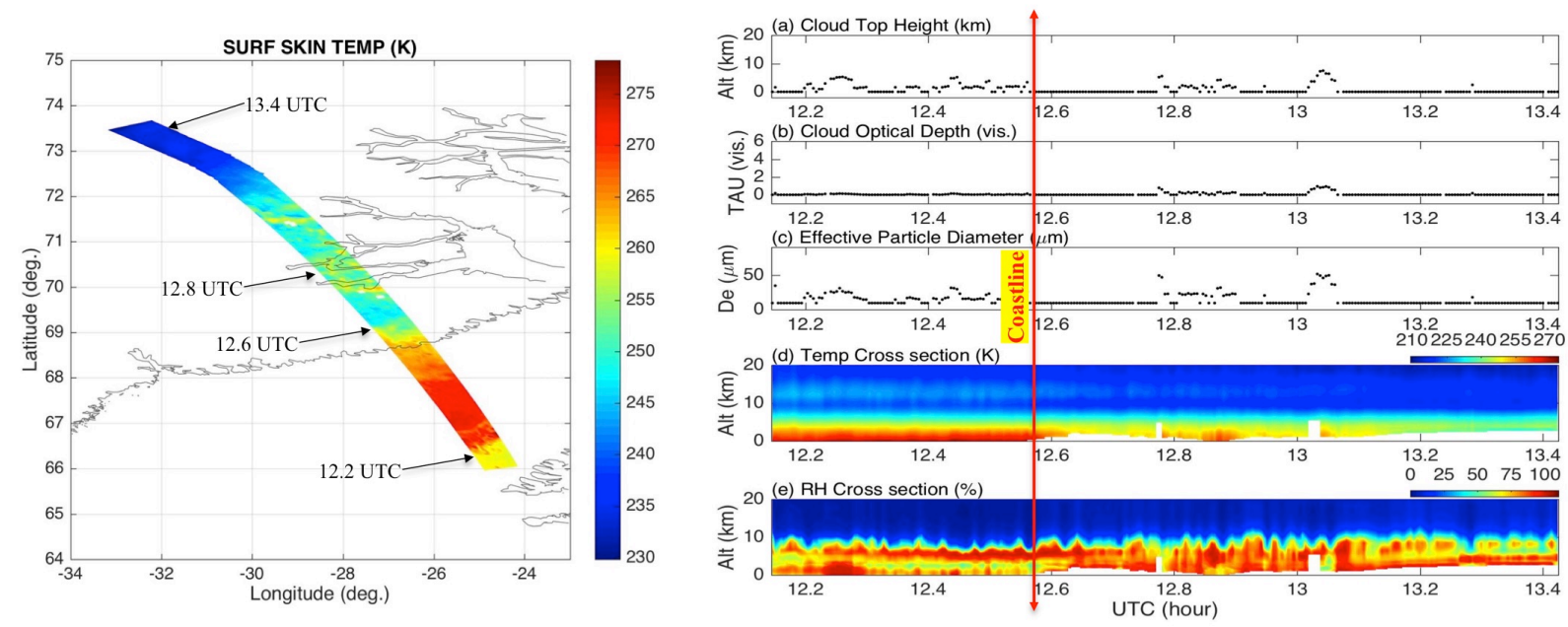

Figure 1. From $23^{\text {rd }}$ March 2015 flight, NAST-I higher resolution retrieval samples (i.e., 2.5-km footprint) indicating huge variations of atmospheric profile and surface skin temperature over the region of interested for this experiment. 
During this experiment, the ER-2 flew under the MetOp-A and -B, Aqua, and Suomi NPP satellites. These satellites carry the IASI, AIRS, and CrIS ultraspectral sounding instruments, respectively. During the campaign, the ER-2 was flown over the Summit Ground Station $\left(72^{\circ} 36^{\prime}\right.$ North latitude, $38^{\circ} 25^{\prime}$ West longitude), a research platform at the summit of the Greenland ice-sheet providing many ground-truth measurements including radiosondes. The primary goals and motivations of this campaign included to employ the ultraspectral sounding technique to validate CrIS radiance measurements (SDRs) and to evaluate atmospheric temperature and moisture profiles (EDRs) retrieved from CrIS/ATMS radiances using similar but different retrieval techniques for aircraft NAST-I radiances in a very cold environment. The results of the CrIS radiance (SDR) validation during this campaign are found elsewhere [17]; here we emphasize the evaluation of retrieved atmospheric state parameters (EDRs). Assuming that CrIS/ATMS retrieval products (EDRs) from the NOAA official operational algorithms (NUCAPS) are to be validated, many validation activities have undergone a search for the "best estimate" atmospheric state. The common approaches in validating retrieved atmospheric states are, for example, to use Numerical Weather Prediction (NWP) model profiles such as those from the ECMWF analysis and "coincident" radiosonde measurements. For this experiment, seven ER-2 science flights $\left(15^{\text {th }}, 19^{\text {th }}, 23^{\text {rd }}, 24^{\text {th }}, 25^{\text {th }}, 28^{\text {th }}\right.$, and $29^{\text {th }}$ of March 2015) were flown under the SNPP satellite and overpassed the Summit Station where the dedicated radiosondes were launched. As NAST-I horizontal resolution is higher than that of the ATMS IFOV $(50-\mathrm{km})$, NAST-I retrievals were averaged within the ATMS IFOVS to compare with CrIS/ATMS NUCAPS retrievals. Shown in Figure 2 are a few profile inter-comparisons between CrIS and NAST-I retrievals together with radiosonde measurements and ECMWF analysis.
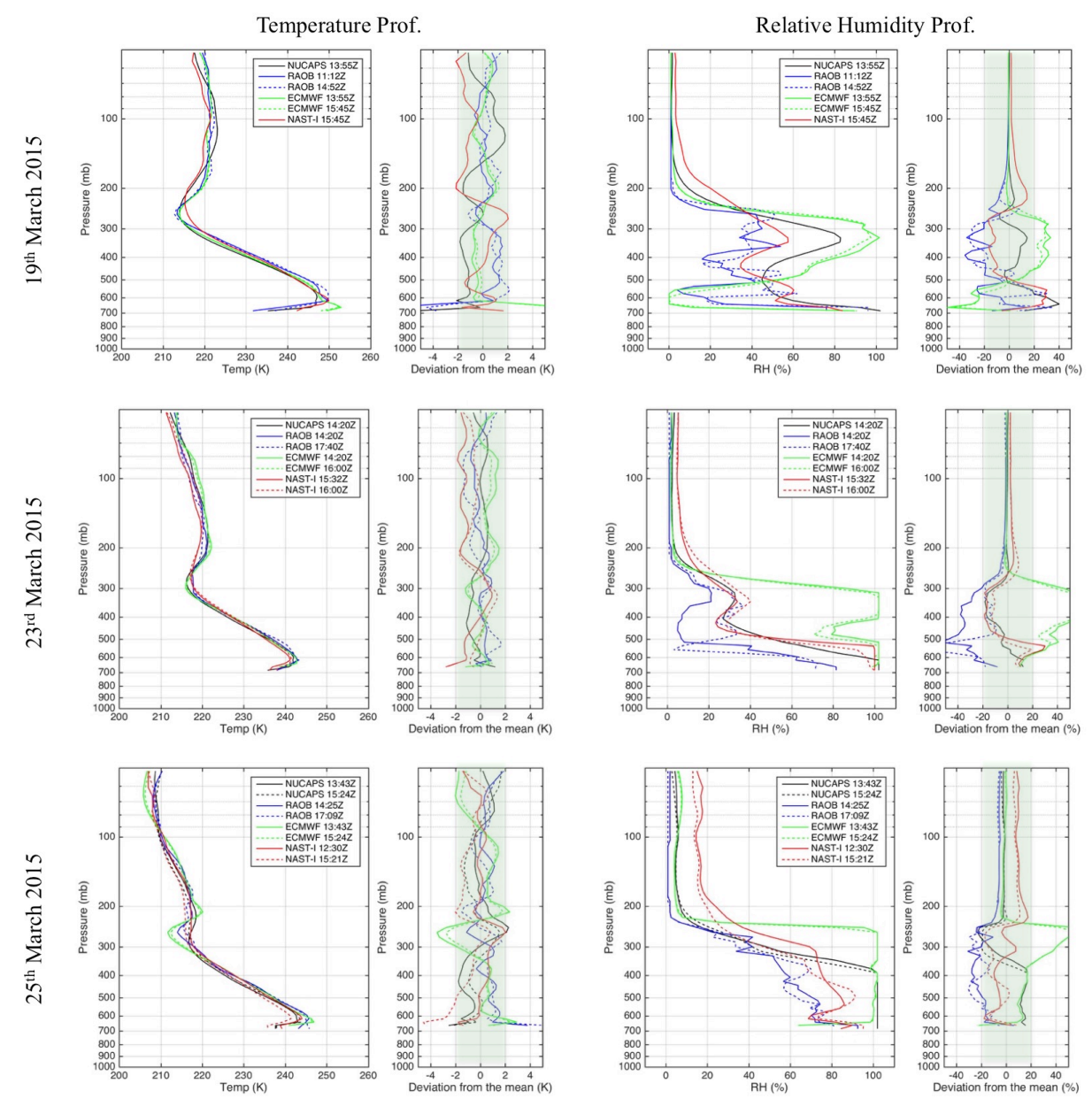

Figure 2. Atmospheric temperature and moisture profile inter-comparison over the Summit for ER-2 flights from $19^{\text {th }}, 23^{\text {rd }}$, and $25^{\text {th }}$ March 2015. NUCAPS is the NOAA official operational algorithm providing CrIS/ATMS retrieval products (EDRs). 
The validation approach for this experiment is to have EDRs from both satellite (CrIS) and aircraft (NAST-I) validated with dedicated but limited radiosondes over the Summit Station together with ECMWF analyses. After the confidence of NAST-I retrieval accuracy using the radiosonde validation is gained, NAST-I retrievals are used to validate satellite CrIS retrievals along the aircraft tracks. The atmospheric profile inter-comparison over the Summit Ground Station was performed for seven science flights; Figure 2 shows some of them. In general, the temperature profiles agree within $\pm 2^{\circ} \mathrm{K}$ and relative humidity being within $\pm 20 \%$ from peak-to-peak between NAST-I and NUCAPS, in term of the deviation from the mean profile. It is worth pointing out that PBL temperature inversion and day-to-day variation of atmospheric thermal properties were captured by both CrIS and NAST-I retrievals and verified with radiosonde measurements and ECMWF analyses.

Using dedicated radiosondes and associated ECMWF profiles at the Summit, a statistical analysis has been performed using ECMWF and radiosondes as a reference, respectively for both satellite CrIS (NUCAPS) and aircraft NAST-I retrievals, shown in Figures 3(a) and 3(b). It is noticed that NUCAPS and NAST-I retrievals were verified against ECMWF for both temperature and relative humidity as shown in Figure 3(a), showing a very similar behavior. In other words, ECMWF is rather deviated from both CrIS and NAST-I retrievals. They do better with radiosondes as shown in Figure 3(b), indicating CrIS and NAST-I retrievals are very similar. Both CrIS and NAST-I have their standard deviation error within $1.5^{\circ} \mathrm{K}$ for temperature and $20 \%$ for relative humidity when radiosonde is assumed as the "truth" profile. Figure 3(c) shows that CrIS retrieval verifies against NAST-I (using NAST-I retrieval as a reference), the standard deviation error is also within $1.5^{\circ} \mathrm{K}$ for temperature and $20 \%$ for relative humidity.

The CrIS/ATMS retrieved atmospheric vertical temperature profile (AVTP) requirement is $1.5^{\circ} \mathrm{K}$ in the region of 3-21 $\mathrm{km}$, and $1.6^{\circ} \mathrm{K}$ in the region of $3 \mathrm{~km}$ and below; retrieved atmospheric vertical moisture profile (AVMP) requirement is $35 \%$ in the region of $4-9 \mathrm{~km}, 20 \%$ in the region of $4 \mathrm{~km}$ and below [7]. We are confident that CrIS retrievals satisfy the requirements when radiosonde and/or NAST-I retrievals is assumed as the "truth" profile, at this Summit location with limited profile data used for this analysis.

(a) Using ECMWF as a reference

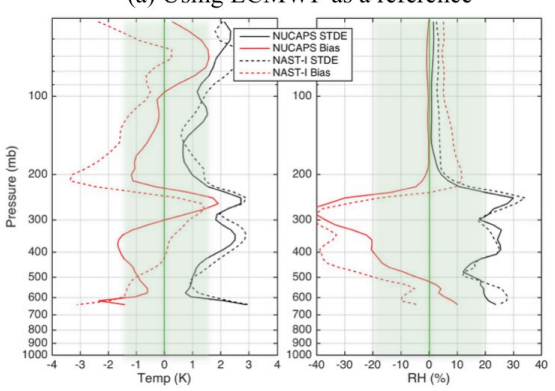

(b) Using RAOB as a reference

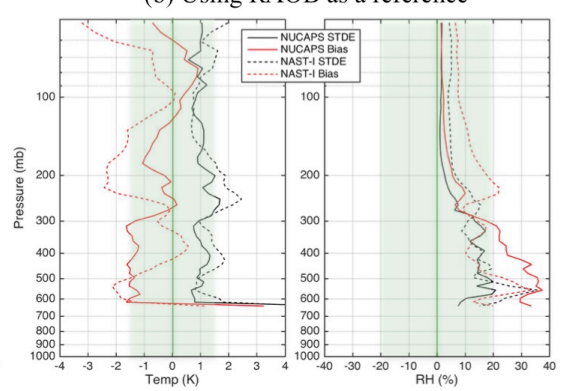

(c) Using NAST-I retrieval as a reference

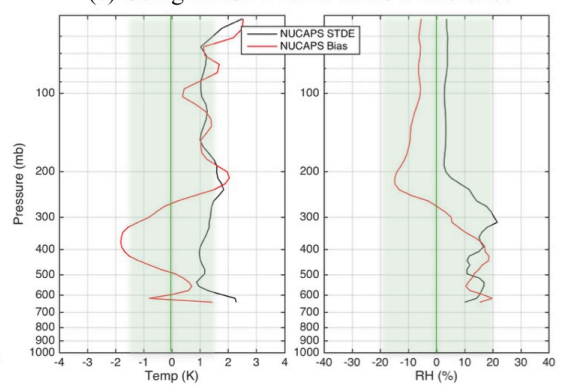

Figure 3. Statistical analysis results based on 11 data samples at the Summit: (a) and (b) CrIS and NAST-I retrievals are verified against ECMWF and radiosonde, respectively, and (c) CrIS retrievals are verified against NAST-I retrieved profiles.

Based on this statistical analysis over the Summit with radiosonde measurements and ECMWF analyses, NUCAPS retrieval STDE relative to radiosonde measurements shown in Figure 3(b) is very similar to that relative to NAST-I retrievals shown in Figure 3(c). Aircraft NAST-I and satellite CrIS retrievals are reconciled within their uncertainties. This implies that NAST-I retrievals are accurate enough to be used to validate CrIS NUCAPS retrievals in the locations where radiosondes are not available. Now we can expand our comparisons to ER-2 tracks over Greenland under SNPP within a temporal window within \pm 2 hours where CrIS and NAST-I are co-located for retrieval inter-comparisons. These co-located measurements are plotted in Figure 4(a) from all seven ER-2 science-flights under clear-sky conditions. A total of 144 samples are used for statistical analysis to derive CrIS NUCAPS STDE relative to NAST-I retrievals, plotted in Figure 4(b). In general, the temperature profile STDE is less than $1.5^{\circ} \mathrm{K}$ and relative humidity STDE is less than $20 \%$. Despite having different radiance measurements from satellite and aircraft instruments and different retrieval algorithms, the two sets of retrievals are in reasonable agreement with each other, indicating that the CrIS NUCAPS 
retrieval accuracy seems to meet the Suomi NPP EDR requirements based upon this Cal/Val campaign over Greenland for a winter, Arctic environment.

(a) Sample Locations

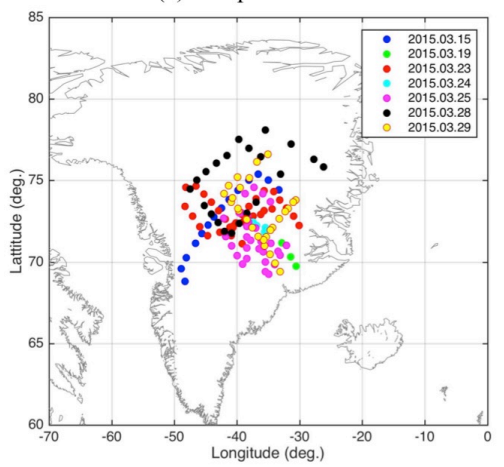

(b) Using NAST-I retrieval as a reference

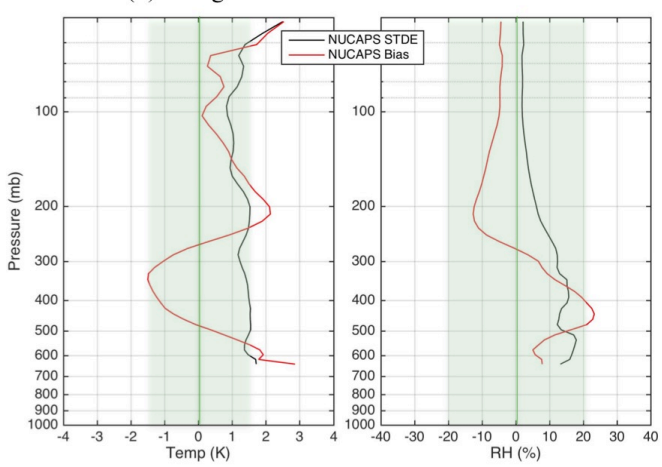

Figure 4. (a) A total of 144 data sample locations used for statistical analysis, (b) CrIS retrievals are verified against NAST-I retrieved profiles.

\section{SUMMARY}

The second SNPP aircraft Cal/Val campaign was successfully conducted with flights over Greenland within a winter Arctic environment; data collected during this campaign have undergone preliminary evaluation and further analyses are ongoing. To understand geophysical information retrieved from satellite observations, such as the Suomi NPP EDRs, validation studies are critical to understanding both the utility of the retrieved data and the performance of the retrieval system. The aircraft program provides such a satellite validation tool because of its similar radiometric observation characteristics and the retrieval systems employed to analyze its data. The NAST-I retrievals, together with additional insitu data collected during airborne field campaigns, are ideal for satellite EDR product validation. In this report, NAST-I retrievals have once again been used to demonstrate their value as an independent, indirect approach to both the evaluation of different retrieval systems and the accuracy of the geophysical state under assessment. NAST-I retrievals obtained using an independent retrieval methodology, together with ECMWF and available radiosonde measurements, are compared with CrIS/ATMS retrievals to give a unique perspective on assessing Suomi NPP EDR performance.

In spite of profile characteristic differences between ECMWF and retrievals, it is shown that the CrIS/ATMS retrieval accuracy seems to satisfy the Suomi NPP EDR requirements based upon data obtained from this airborne Cal/Val campaign conducted over Greenland within a winter, Arctic atmosphere and environment. Limited data analysis from this second Suomi NPP field campaign might not statistically reflect an all-seasonal-global Suomi NPP EDR accuracy, however, each comparison of individual retrievals is critical to understanding retrieval performance under specific atmospheric conditions. In order to validate satellite EDR performance under a variety of atmospheric conditions, it is recommended that aircraft field campaigns be conducted in different geophysical locations for different atmospheric and surface environments and that these be repeated periodically to ensure long-term measurement performance confidence over the life of the satellite program.

Acknowledgements. The authors greatly appreciate the contributions of NASA's Langley Research Center and the UK Met Office. The authors are also thankful for dedicated NASA ER-2 aircraft pilots and crewmembers based at NASA's Armstrong Flight Research Center. The NAST-I program is supported by NASA Headquarters, NASA Langley Research Center, and NOAA NESDIS/JPSS Program Office. In addition, the authors wish to specifically acknowledge Dr. Jack Kaye of NASA's Science Mission Directorate for his continued, enabling support of the NAST-I program. 


\section{REFERENCES}

[1] Cousins, D. and W. L. Smith, "National Polar-Orbiting Operational Environmental Satellite System (NPOESS) Airborne Sounder Testbed-Interferometer (NAST-I)," Proc. SPIE, vol. 3127, pp. 323-331 (1997).

[2] Aumann, H. H., M. T. Chahine, C. Gautier, M. D. Goldberg, E. Kalnay, M. L. McMillin, H. Revercomb, P. W. Rosenkranz, W. L. Smith, D. H. Staelin, L. L. Strow, and J. Susskind, "AIRS/AMSU/HSB on the Aqua mission: design, science objective, data products, and processing systems," IEEE Trans. Geosci. Remote Sensing, vol. 41, pp. 253-264 (2003).

[3] Pagano, T. S., H. H. Aumann, D. E. Hagan, and K. Overoye, "Prelaunch and in-flight radiometric calibration of the Atmospheric Infrared Sounder (AIRS)," IEEE Trans. Geosci. Remote Sensing, vol. 41, pp. 265-273, (2003).

[4] Blumstein, D., G. Chalon, T. Carlier, C. Buil, P. Hebert, T. Maciaszek, G. Ponce, and T. Phulpin, "IASI instrument: Technical overview and measured performances," Proc. SPIE, vol. 5543, pp. 196-207 (2004).

[5] Klaes, D. K., M. Cohen, Y. Buhler, P. Schlüssel, R. Munro, J.-P. Luntama, A. VonEngeln, E.Ó. Clerigh, H. Bonekamp, J. Ackermann, and J. Schmetz, "An introduction to the EUMETSAT polar system," Bull. Amer. Meteorol. Soc., vol. 88, no. 7, pp. 1085-1096 (2007).

[6] Bloom, H. J., "The cross-track infrared sounder (CrIS): a sensor for operational meteorological remote sensing," Proc. IGARSS, vol. 3, pp. 1341-1343 (2001).

[7] Divakarla, M., B. Barnet, X. Liu, D. Gu, M. Wilson, S. Kizer, X. Xiong, E. Maddy, R. Ferraro, R. Knuteson, D. Hagan, X.-L. Ma, C. Tan, N. Nalli, A. Reale, A. K. Mollner, W. Yang, A. Gambacorta, M. Feltz, F. IturbideSanchez, B. Sun, and M. Goldberg, "The CrIMSS EDR algorithm: characterization, optimization, and validation," J. Geophys. Res., vol. 119, no. D8, pp. 4953-4977. (2014).

[8] Gambacorta, A., C. Barnet, W. Wolf, T. King, N. Nalli, M. Wilson, L. Soulliard, K. Zang, X. Xiong, and M. Goldberg, "The NOAA operational hyper spectral retrieval algorithm: a cross-comparison among the CrIS, IASI and AIRS processing system," Proc. International TOVS Study Conference, Jeju Island, South Korea (2014).

[9] Taylor, J. P., W. L. Smith, V. Cuomo, A. M. Larar, D. K. Zhou, C. Serio, T. Maestri, R. Rizzi, S. Newman, P. Antonelli, S. Mango, P. Di Girolamo, F. Esposito, G. Grieco, D. Summa, R. Restieri, G. Masiello, F. Romano, G. Pappalardo, G. Pavese, L. Mona, A. Amodeo, and G. Pisani, "EAQUATE - An international experiment for hyper-spectral atmospheric sounding validation," Bull. Amer. Meteor. Soc., vol. 89, no. 2, pp. 203-218 (2008).

[10] Zhou, D. K., W. L. Smith, V. Cuomo, J. P. Taylor, C. D. Barnet, P. Di Girolamo, G. Pappalardo, A. M. Larar, X. Liu, S. M. Newman, C. Lee, and S. A. Mango, "Retrieval validation during the European aqua thermodynamic experiment," Q. J. R. Meteorol. Soc., vol. 133, no. S3, pp. 203-215 (2007).

[11] Liu, X., D. K. Zhou, A. M. Larar, W. L. Smith, and S. A. Mango, "Case study of a principal-component-based radiative transfer forward model and retrieval algorithm using EAQUATE data," Q. J. R. Meteorol. Soc., vol. 133, no. S3, pp. 243-256 (2007).

[12] Larar, A. M., W. L. Smith, D. K. Zhou, X. Liu, H. Revercomb, J. P. Taylor, S. M. Newman, and P. Schlüssel, "IASI spectral radiance validation inter-comparisons: case study assessment from the JAIVEx field campaign," Atmos. Chem. Phys., vol. 10, no. 2, pp. 441-430 (2010).

[13] Liu, X., D. K. Zhou, A. M. Larar, W. L. Smith, P. Schlüssel, S. M. Newman, J. P. Taylor, and W. Wu, "Retrieval of atmospheric profiles and cloud properties from IASI spectra using super-channels," Atmos. Chem. Phys., vol. 9, no. 23, pp. 9121-9142 (2009).

[14] Zhou, D. K., W. L. Smith, A. M. Larar, X. Liu, J. P. Taylor, P. Schlüssel, L. L. Strow, and S. A. Mango, "All weather IASI single field-of-view retrievals: Case study-Validation with JAIVEx data," Atmos. Chem. Phys., vol. 9, no. 15, pp. 2241-2255 (2009).

[15] Zhou, D. K., X. Liu, A. M. Larar, J. Tian, W. L. Smith, W. Wu, S. H. Kizer, Q. Liu, and M. D. Goldberg, "First Suomi NPP Cal/Val Campaign: Inter-comparison of Satellite and Aircraft Sounding Retrievals," IEEE JSTARS, in print (2016)

[16] Zhou, D. K., A. M. Larar, X. Liu, W. L. Smith, Sr., and L. L. Strow, "Error Consistency Analysis Scheme for ultraspectral sounding retrieval error budget estimation," Remote Sens. Lett., vol. 4, no. 3, 219-227 (2013).

[17] Larar, A. M., D. K. Zhou, X. Liu, J. Tian, and W. L. Smith, "Advanced sounder validation studies from recent NAST-I airborne field campaigns," Proc. SPIE 9880, this issue, in print (2016). 